\title{
MEAN VALUE
}

THEOREMS

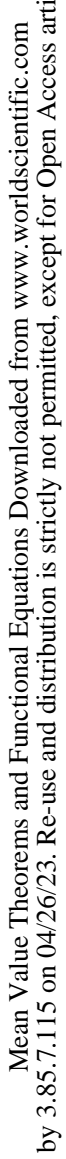

\author{
AND \\ FUNCTIONAL
}

EQUATIONS 
This page is intentionally left blank 


\section{MEAN VALUE}

\section{THEOREMS}

\section{AND}

FUNCTIONAL

\section{EQUATIONS}

\section{P. K. Sahoo \\ T. Riedel}

University of Lovisville

Lovisville, KY, USA 


\section{Published by}

World Scientific Publishing Co. Pte. Ltd.

P O Box 128, Farrer Road, Singapore 912805

USA office: Suite 1B, 1060 Main Street, River Edge, NJ 07661

UK office: 57 Shelton Street, Covent Garden, London WC2H 9HE

\section{British Library Cataloguing-in-Publication Data}

A catalogue record for this book is available from the British Library.

\section{MEAN VALUE THEOREMS AND FUNCTIONAL EQUATIONS}

Copyright (C) 1998 by World Scientific Publishing Co. Pte. Ltd.

All rights reserved. This book, or parts thereof, may not be reproduced in any form or by any means, electronic or mechanical, including photocopying, recording or any information storage and retrieval system now known or to be invented, without written permission from the Publisher.

For photocopying of material in this volume, please pay a copying fee through the Copyright Clearance Center, Inc., 222 Rosewood Drive, Danvers, MA 01923, USA. In this case permission to photocopy is not required from the publisher.

ISBN 981-02-3544-5

This book is printed on acid-free paper.

Printed in Singapore by Uto-Print 
To

Janos Aczél,

Palaniappan Kannappan and Berthold Schweizer

from whom we have learned so much about functional equations

and

to our families

Prasanna Sahoo

Thomas Riedel 
This page is intentionally left blank 


\section{Preface}

Lagrange's mean value theorem is a very important result in analysis. It originated from Rolle's theorem, which was proved by the French mathematician Michel Rolle (1652-1719) for polynomials in 1691. This theorem appeared for the first time in the book Methode pour resoudre les égalitez without a proof and without any special emphasis. Rolle's theorem got its recognition when Joseph Lagrange (1736-1813) presented his mean value theorem in his book Theorie des functions analytiques in 1797 . It received further recognition when Augustin Louis Cauchy (1789-1857) proved his mean value theorem in his book Equationnes differentielles ordinaires. Most of the results in Cauchy's book were established using the mean value theorem or indirectly Rolle's theorem. Since the discovery of Rolle's theorem (or Lagrange's mean value theorem), many papers have appeared directly or indirectly dealing with Rolle's theorem. Recently, many functional equations were studied motivated by various mean value theorems, and the main goal of this book is to present some results related to the mean value theorem (MVT) and its generalizations and many of these functional equations. This book started out as lecture notes for a seminar on mean value theorems and related functional equations held at the University of Louisville. The intent was to introduce advanced undergraduate and beginning graduate students to functional equations and to introduce those who had not yet taken a course in mathematical analysis to the basics of reading and writing proofs. We found that the mean value theorem with all it's generalizations and related functional equations was the ideal theme, a vast amount of material not usually included in a standard curriculum could be covered without spending large amounts of time on prerequisites. 
Of course, we do not claim that this book covers everything related to the mean value theorem, there are many results we do not cover because they lie outside the scope of this book. Also, all functions appearing in the functional equations treated in this book are real or complex valued; we did not cover any functional equation where the unknown functions take on values on an algebraic structure such as group, ring or field. Our primary reason for writing this book is to introduce to our reader the simplicity and beauty of functional equations on one hand and the importance of the mean value theorem on the other hand. Almost all functional equations treated in this book are very elementary. The innovation of solving these functional equations lies in finding the right tricks for a particular case. One should not be misled by the elementary nature of the functional equations. discussed in this book. We have stated many open problems related to some simple looking functional equations. It looks as though anyone could solve them, but, to the best of our knowledge, nobody has succeeded in finding the general solutions of these equations without any regularity conditions on the unknown functions.

We now give a brief description of the contents. Chapter 1 gives an account of additive and biadditive functions. In this chapter, we treat the Cauchy functional equation and show that continuous or locally integrable additive functions are linear. We further explore the behavior of discontinuous additive functions and show that they display a very strange behavior: their graphs are dense in the plane. To this end, we briefly discuss the Hamel basis and it's use for constructing discontinuous additive functions. Additive functions on the real and complex planes are also treated in this chapter and, finally, we give a brief exposition on biadditive functions. This chapter concludes with a discussion of open problems.

In Chapter 2, Lagrange's mean value theorem is treated. Many examples are given to illustrate its importance. The mean value theorem is the motivation for many functional equations, some of which are treated in this chapter. Many of these results on functional equations are very recent. Further, we briefly describe the mean value theorem for divided differences and give some applications in defining the functional means. In this chapter we also examine the limiting behavior of the mean values. This chapter also concludes with a discussion of open problems.

Chapter 3 deals with a variation of Lagrange's mean value theorem due to Dimitri Pompeiu. Pompeiu's mean value theorem has been the source of motivations for many Stamate type functional equations. In this 
chapter, we discuss several of them as well as a functional equation due to Marec Kuczma. We have simplified his proof by making some additional assumptions. This chapter examines also some functional equations which are extensions of previously studied equations and many are motivated by Simpson's rule for numerical integration. This chapter also concludes with a discussion of open problems.

In Chapter 4, we examine and extend Lagrange's mean value theorem to functions in two variables. We also discuss some functional equations that arise from such an extension. Some generalized mean value type functional equations and Cauchy's mean value theorem for functions in two variables are treated in this chapter as well. This chapter also concludes with a discussion of open problems.

Chapter 5 focuses on various generalizations of Lagrange's mean value theorem. We first treat all generalizations of the mean value theorem for functions from reals into reals. In this chapter, we examine various generalizations due to Flett, Trahan and many other mathematicians. We further treat mean value theorems for real valued functions on the plane, and present some results due to Clarke and Ledyaev. Mean value theorems for vector valued functions on reals are also treated in this chapter including results of McLeod and Sanderson. The mean value theorem for vector valued functions on the plane is also covered. A recent result of Furi and Martelli is included here and we also treat the mean value theorem for complex valued function on the complex plane. This chapter concludes with a conjecture due to Furi and Martelli and its recent proof by Ferrer.

Chapter 6 examines the mean value theorem and its generalizations for function with symmetric derivatives and Dini derivatives, respectively. Here, we introduce the notion of symmetric differentiation and then derive the mean value theorem for symmetrically differentiable functions. The notion of Dini derivatives is introduced with some well known examples. Finally, we present the mean value theorem for nondifferentiable functions.

Chapter 7 deals with the integral mean value theorem and its generalizations. Some applications of it are given, such as finding the integral representations of the arithmetic, geometric, logarithmic, and identric means and their extensions. Here we also discuss the iterations of arithmetic and geometric means and a theorem due to Kranz and Thews which states that if the mean values from the integral mean value theorem and differential mean value theorem occur at the same point then the underlying function is exponentially affine. This chapter concludes with some open problems. 
This book would not have been completed without the support and encouragement from Robert Powers. He read a part of the manuscript and made many constructive criticisms for improvement. We are very grateful to him. We are also thankful to Elias Deeba for his many suggestions for improvement. We express our gratitude to Mario Martelli for providing us some valuable references and reprints of his papers related to Rolle's theorem. We are extremely thankful to Jürg Rätz for bringing our attention to his work on Cauchy's mean value theorem for divided differences. In this book, we have used results from many researchers and we have made honest efforts to pay credit to appropriate researchers. If we have missed anyone, we are sorry. If there is any resemblance with any published proof to a proof in this book it is because we could not improve the original proof of the author. We thank students who regularly attended our seminar on mean value theorems and functional equations, where the idea for writing this book was conceived. While this book was in its final form of revision, the first author visited a number of universities including the Technical University of Braunschweig (Germany), the University of Waterloo (Canada), the Aizu University (Japan), the Indian Statistical Institute (India) and the Sambalpur University (India) during his sabbatical in the Fall term of 1995. He would like to thank these universities for providing him with excellent work environment and hospitalities.

This book was typeset by authors in $\mathrm{AAT}_{\mathrm{E}} \mathrm{X}$, a macro package written by Leslie Lamport for Donald Knuth's TEX typesetting package. The bibliography and index were compiled using BibTeX and MakeIndex, respectively.

Prasanna Sahoo

Thomas Riedel 


\section{Contents}

Preface vii

Chapter 1 Additive and Biadditive Functions 1

1.1 Continuous Additive Functions . . . . . . . . . . . . . 1

1.2 Discontinuous Additive Functions . . . . . . . . . . . . 6

1.3 Other Criteria for Linearity . . . . . . . . . . . . . . . . . . . 11

1.4 Additive Functions on the Real and Complex Plane . . . . . . 12

1.5 Biadditive Functions . . . . . . . . . . . . . . . 18

1.6 Some Open Problems . . . . . . . . . . . . . . . 22

Chapter 2 Lagrange's Mean Value Theorem and Related Functional Equations $\quad 25$

2.1 Lagrange's Mean Value Theorem . . . . . . . . . . . . . . . 25

2.2 Applications of the MVT . . . . . . . . . . . 30

2.3 Associated Functional Equations . . . . . . . . . . . . . . 40

2.4 The MVT for Divided Differences . . . . . . . . . . . . . 58

2.5 Limiting Behavior of Mean Values . . . . . . . . . . . . 64

2.6 Cauchy's MVT and Functional Equations . . . . . . . . 77

2.7 Some Open Problems . . . . . . . . . . . . . . 80

Chapter 3 Pompeiu's Mean Value Theorem and Associated $\begin{array}{ll}\text { Functional Equations } & \mathbf{8 3}\end{array}$

3.1 Pompeiu's Mean Value Theorem . . . . . . . . . . . . . . . 83

3.2 Stamate Type Equations . . . . . . . . . . . . . . . . . . . . . . . . . . . . . . 85

3.3 An Equation of Kuczma . . . . . . . . . . . . . . . . 92 
3.4 Equations Motivated by Simpson's Rule . . . . . . . . . . . . 98

3.5 Some Generalizations . . . . . . . . . . . . . . . . . . . . . . 108

3.6 Some Open Problems . . . . . . . . . . . . . . . . . 125

Chapter 4 Two-dimensional Mean Value Theorems and $\begin{array}{ll}\text { Functional Equations } & 127\end{array}$

4.1 MVTs for Functions in Two Variables . . . . . . . . . . . . . 127

4.2 Mean Value Type Functional Equations . . . . . . . . . . . . 129

4.3 Generalized Mean Value Type Equations . . . . . . . . . . . . 135

4.4 Cauchy's MVT for Functions in Two Variables . . . . . . . . . 144

4.5 Some Open Problems . . . . . . . . . . . . . . . . . . . . . . 144

Chapter 5 Some Generalizations of Lagrange's Mean Value $\begin{array}{ll}\text { Theorem } & 147\end{array}$

5.1 MVTs for Real Functions . . . . . . . . . . . . . . . 147

5.2 MVTs for Real Valued Functions on the Plane . . . . . . . . 156

5.3 MVTs for Vector Valued Functions on the Reals . . . . . . . . 160

5.4 MVTs for Vector Valued Functions on the Plane . . . . . . . 163

5.5 MVTs for Functions on the Complex Plane . . . . . . . . . . 168

5.6 A Conjecture of Furi and Martelli . . . . . . . . . . . . . 180

Chapter 6 Mean Value Theorems for Some Generalized Derivatives

6.1 Symmetric Differentiation of Real Functions . . . . . . . . . . . 181

6.2 A Quasi-Mean Value Theorem . . . . . . . . . . . . . . . . 188

6.3 An Application . . . . . . . . . . . . . . . . . . . . . . . . 192

6.4 Generalizations of MVTs . . . . . . . . . . . . . 193

6.5 Dini Derivatives of Real Functions . . . . . . . . . . . . 195

6.6 MVTs for Nondifferentiable Functions . . . . . . . . . . . . . 201

Chapter 7 Some Integral Mean Value Theorems and Related Topics 207

7.1 The Integral MVT and Generalizations . . . . . . . . . . 207

7.2 Integral Representation of Means . . . . . . . . . . . . . . 218

7.3 Coincidence of Mean Values . . . . . . . . . . . . . . . . . 224

7.4 Some Open Problems . . . . . . . . . . . . . . . . 227

Bibliography 233

Index 